\title{
Actividad bioquímica y molecular de enzimas del estrés oxidativo en plantas de
} tomate creciendo con plomo

\section{Biochemical and molecular activity of oxidative stress enzymes in tomato plants growth with lead}

\section{Sandra Pérez-Àlvarez $1 *$ (D) \\ Eduardo Fidel Héctor-Ardisana2 2 (D) \\ Cesar Marcial Escobedo-Bonilla 3 \\ María Antonia Flores-Córdova 4 (1) \\ Esteban Sánchez-Chávez 5 (D) \\ Crescencio Urias Garcia ${ }^{1}$ [D] \\ ${ }^{1}$ Facultad de Ciencias Agrícolas y Forestales, Universidad Autónoma de Chihuahua, $\mathrm{Km} \mathrm{2.5,} \mathrm{carretera}$ Delicias-Rosales, campus Delicias, CP. 33000 . CD. Delicias, Chihuahua, México. \\ ${ }^{2}$ Instituto de Posgrado, Universidad Técnica de Manabí, CP. EC13010. Portoviejo, Ecuador. \\ ${ }^{3}$ Instituto Politécnico Nacional-CIIDIR Unidad Sinaloa, Juan de Dios Bá- tiz Paredes No. 250, CP. 81101 Guasave, Sinaloa, México. \\ ${ }^{4}$ Facultad de Ciencias Agrotec- nológicas Universidad Autónoma de Chihuahua. Av. Pascual Orozco s/n, Campus 1, CP. 31000. Santo Niño, Chihuahua, Chihuahua, México. \\ ${ }^{5}$ Centro de Investigación en Ali- mentación y Desarrollo A.C. Unidad Delicias, Av. Cuarta Sur 3828, Fracc. Vencedores del Desierto, CP. 33089. Delicias, Chihuahua, México. \\ *Autor de correspondencia: spalvarez@uach.mx}

\section{Nota científica}

Recibida: 22 de octubre 2021 Aceptada: 07 de diciembre 2021

Como citar: Pérez-Àlvarez S, HéctorArdisana EF, Escobedo-Bonilla CM, Flores-Córdova MA, Sánchez-Chávez E, Urías García C (2021) Actividad bioquímica y molecular de enzimas del estrés oxidativo en plantas de tomate creciendo con plomo. Ecosistemas y Recursos Agropecuarios 8(3): e3163. DOI: 10.19136/era.a8n3.3163
RESUMEN. El objetivo de esta investigación fue estudiar la actividad a nivel bioquímico y molecular de enzimas del estrés oxidativo en plantas de tomate cv. Mariela cultivadas con diferentes concentraciones de plomo $(0,50 \mathrm{y}$ $100 \mathrm{mg} \mathrm{kg}^{-1}$ de $\mathrm{PbAc}_{2}$ ). Para la actividad bioquímica, se seleccionaron las enzimas superóxido dismutasa (SOD) y glutatión reductasa (GR); además, se determinó la concentración de proteínas. La actividad molecular se estudió mediante PCR en tiempo real para tres genes: SOD, isoflavona reductasa (IFR) y proteína tumoral controlada transcripcionalmente (TCTP). En hojas y tallos hubo diferencias significativas en las proteínas comparadas con el control. La GR mostró que en hojas la mayor actividad se logró a 50 $\mathrm{mg} \mathrm{kg}^{-1}$ de $\mathrm{PbAc}_{2}$, mientras que en tallos hubo diferencias con el control. En hojas y tallos la menor actividad de la SOD fue al utilizar $\mathrm{PbAc}_{2}$. La expresión de los tres genes en hojas fue mayor con $\mathrm{PbAc}_{2}$ y en tallos varió.

Palabras clave: Contaminación del suelo, estrés oxidativo, metales pesados, Solanum lycopersicum L., toxicidad vegetal.

ABSTRACT. The aim of this research was to study the activity at biochemical and molecular level of oxidative stress enzymes in tomato plants cv. Mariela grown with different concentrations of lead $\left(0,50\right.$ and $100 \mathrm{mg} \mathrm{kg}^{-1}$ of $\mathrm{PbAc}_{2}$ ). For the biochemical activity, the enzymes superoxide dismutase (SOD) and glutathione reductase (GR) were selected; in addition, the protein concentration was determined. Molecular activity was studied by real-time PCR for three genes: SOD, isoflavone reductase (IFR), and transcriptionally controlled tumor protein (TCTP). In leaves and stems there were significant differences in proteins compared to the control. The GR showed that in leaves the highest activity was achieved at $50 \mathrm{mg} \mathrm{kg}^{-1}$ of $\mathrm{PbAc}_{2}$, while in stems there were differences with the control. In leaves and stems, the lowest $\mathrm{SOD}$ activity was when using $\mathrm{PbAc}_{2}$. The expression of the three genes in leaves was higher with $\mathrm{PbAc}_{2}$ and in stems it varied.

Key words: Soil contamination, oxidative stress, heavy metals, Solanum lycopersicum L., plant toxicity. 


\section{INTRODUCCIÓN}

La producción mundial de tomate (Solanum lycopersicum L.) en 2019 fue de 180766329 t (FAOSTAT 2021) y es el segundo cultivo de hortalizas más importante a nivel mundial después de la papa (Costa y Heuvelink 2018). Con respecto a la variedad Mariela de tomate, tiene altos rendimientos, frutos grandes y un comportamiento adecuado frente a los virus, Alternaria solani (Cooke) Wint. y Phytophthora infestans (Mont.) de Bary (Moya et al. 2000). De acuerdo con Al-Qurainy (2009) hay aproximadamente 400 especies de plantas de al menos 45 familias que son hiperacumuladoras de metales. Con respecto al tomate, es una planta que se ha utilizado en algunos estudios como planta hiperacumuladora, para la descontaminación de suelos con alta concentración de metales (Kumar et al. 2009, Bizily et al. 1999), ya que acumula hasta alrededor de $100 \mathrm{mg}$ $\mathrm{kg}^{-1}$ peso seco de $\mathrm{Cd}$, hasta $1000 \mathrm{mg} \mathrm{kg}^{-1}$ peso seco de $\mathrm{Ni}, \mathrm{Cu}, \mathrm{Co}, \mathrm{Cr}$ y Pb, y hasta $10000 \mathrm{mg} \mathrm{kg}^{-1}$ peso seco de Zn y Mn (Bizily et al. 1999).

El plomo $(\mathrm{Pb})$ es uno de los metales pesados más tóxicos incluso en concentraciones muy bajas que puede acumularse en diferentes partes induciendo la inhibición del crecimiento de las raíces, crecimiento subdesarrollado, ennegrecimiento del sistema radicular y clorosis (Sharma y Dubey 2005). Al respecto, Akinci et al. (2010) explica que en plantas de tomate la elongación de la raíz y altura de la planta; biomasa fresca y seca de raíces, brotes y hojas; área foliar fueron negativamente afectadas por el aumento de las concentraciones de plomo $(75,150$ and $300 \mathrm{mg} \mathrm{L}^{-1}$ ).

El sistema que regula a las especies reactivas de oxígeno (EROs) para mantenerlas a un nivel no dañino en las células vegetales incluye una red de antioxidantes no enzimática y enzimática (Sachdev et al. 2021), esta última incluye enzimas como la superóxido dismutasa (SOD), glutatión reductasa (GR), catalasa (CAT), entre otras (Hasanuzzaman et al. 2012, Kaur et al. 2019). Teniendo en cuenta que el nivel de metales pesados en los suelos agrícolas au- menta año tras año y que el tomate es una hortaliza muy consumida en el mundo, además del poco conocimiento que existe acerca de las respuestas de las enzimas del estrés oxidativo a nivel bioquímico y molecular en plantas de tomate que crecen en suelos contaminados con plomo, el objetivo de este trabajo fue analizar la actividad de algunas enzimas del estrés oxidativo a nivel bioquímico y molecular en plantas de tomate. cv. Mariela con diferentes concentraciones de $\mathrm{PbAc}_{2}$, lo que permitirá una mejor comprensión de esas respuestas en este cultivo.

\section{MATERIALES Y MÉTODOS}

Las semillas de tomate cv. Mariela se sembraron en bandejas que contenían una mezcla 1: 1 ( $\mathrm{v}$ / v) de vermiculita suplementada con NPK (nitrógeno, fósforo y potasio) 10:10:10 (g) y un producto comercial (Plantmax HT Eucatex, Sao Paulo, Brasil) al 2\% $(p / v)$ y se colocaron en condiciones de invernadero en la Escuela Superior de Agricultura de la Universidad de Sao Paulo, Brasil. Las plántulas se trasplantaron una vez que aparecieron las primeras hojas verdaderas a macetas de $1 \mathrm{~L}$ (unidad experimental) con arena y tres concentraciones de Acetato de plomo $\left(\mathrm{PbAc}_{2} ; 0,50\right.$ y $\left.100 \mathrm{mg} \mathrm{kg}^{-1}\right)$ con tres repeticiones cada una. Estas concentraciones de plomo se utilizaron considerando que las concentraciones de este metal son 40,70 y $100 \mathrm{mg} \mathrm{kg}^{-1}$ para suelos arenosos, limosos y arcillosos, respectivamente (BMJV 2017).

A las plantas de tomate se les aplicaron 50 $\mathrm{mL}$ de las soluciones de $\mathrm{PbAc}_{2}$ aplicándosele a cada maceta 0,50 o $100 \mathrm{mg} \mathrm{kg}^{-1}$ de $\mathrm{PbAc}_{2}$ dependiendo del tratamiento con una frecuencia de dos veces por semana a partir del día del trasplante hasta el inicio de la fase reproductiva (16 veces en 60 días); cuando las aplicaciones fueron completas, se recolectaron hojas y tallos representando la fase vegetativa de crecimiento. Terminada la recolección, los órganos vegetativos se lavaron con agua desionizada para almacenarse por separado a $-80{ }^{\circ} \mathrm{C}$ hasta su análisis. 


\section{Determinación de la actividad de las enzimas an- tioxidantes}

Para el estudio de la actividad enzimática, las muestras se manejaron a $4{ }^{\circ} \mathrm{C}$. Las muestras de hojas y tallos por separado se homogeneizaron en un mortero con tampón de potasio $100 \mathrm{mM}$ $(\mathrm{pH} 7,5)$ que contenía ácido etilendiaminotetraacético (EDTA) $1 \mathrm{mM}$, DL-ditiotreitol $3 \mathrm{mM}$ y $5 \%(\mathrm{p} / \mathrm{v})$ de polivinilpirrolidona en proporción de 2: 1 (volumen de tampón / peso fresco); luego se centrifugaron 30 min a $10.000 \times \mathrm{g}$ y el sobrenadante se almacenó en alícuotas a $-80{ }^{\circ} \mathrm{C}$ (Azevedo et al. 1998).

La actividad de la GR (EC 1. 8. 1. 7) se analizó por espectrofotocolorimetría a $30{ }^{\circ} \mathrm{C}$ en una mezcla con $3 \mathrm{~mL}$ de tampón de fosfato de potasio $100 \mathrm{mM}$ $(\mathrm{pH}$ 7.5) con ácido nitrobenzoico $1 \mathrm{mM}$, GSSG (disulfuro de glutatión) $1 \mathrm{mM}$, NADPH 0,1 mM (fosfato de dinucleótido de nicotinamida y adenina en forma reducida) y finalmente se añadió el extracto vegetal. El valor de reducir el GSSG se controló mediante el aumento de la absorbancia a $412 \mathrm{~nm}$ durante 2 minutos (Gomes-Junior et al. 2006). La actividad de la GR se determinó en $\mu \mathrm{mol}$ min $\mathrm{mg}^{-1}$ de proteína.

La actividad de la SOD (EC 1.15.1.1) se determinó monitoreando la inhibición de la reacción fotoquímica del nitro azul tetrazolio (NBT) (Beyer y Fridowich 1987), donde una unidad de actividad SOD se definió como la cantidad de enzima necesaria para causar el $50 \%$ de inhibición de la reducción del NBT monitoreada a $560 \mathrm{~nm}$. La actividad de la SOD se midió en $\mu \mathrm{mol}$ min $\mathrm{mg}^{-1}$ de proteína. El análisis de la actividad de las enzimas en estudio se realizó con un espectrofotocolorímetro modelo Ray Leigh UV-2601.

\section{Análisis de la concentración de las proteínas solubles totales}

La concentración de las proteínas solubles totales se analizó por el método de Bradford (1976) con albúmina bovina (BSA) como estándar.

\section{Análisis estadístico}

El diseño experimental utilizado fue bloques al azar con tres concentraciones de $\mathrm{Pb}(0,50$ y 100 $\left.\mathrm{mg} \mathrm{kg} \mathrm{PbAc}_{2}\right)$ y se realizaron tres repeticiones con 10 unidades experimentales en total. Se utilizó un ANOVA de clasificación simple y una prueba de Tukey $(p \leq 0.05)$ para comparar las medias entre los tratamientos. Se utilizó el programa estadístico SAS versión 9.4 .

\section{PCR en Tiempo Real}

Un mini kit de plantas GeneEluteTM (SigmaAldrich ${ }^{\circledR}$ ) se utilizó para la extracción del ARN total. La concentración del ácido nucleico se determinó con un espectrofotómetro NanoDrop (ND-1000, Thermo Scientific, EE. UU.). La síntesis del ADNc se llevó a cabo con el sistema SuperScriptTM (Invitrogen life technologies) y se llevó a cabo una PCR (Thermocycler Applied Systems 2720, EE. UU.) con un ciclo a $42{ }^{\circ} \mathrm{C}$ durante $5 \mathrm{~min}, 50{ }^{\circ} \mathrm{C}$ durante $50 \mathrm{~min}$ y $70{ }^{\circ} \mathrm{C}$ durante $15 \mathrm{~min}$.

Se estudió la expresión de tres genes involucrados en las respuestas a estrés abiótico en cultivos de interés (IFR, TCTP y SOD). La PCR en tiempo real (RT-PCR) se realizó con cebadores que fueron diseñados con el software Primer Express 2.00 (Applied Biosystems) partiendo de secuencias recuperadas de la base de datos $\mathrm{NCBI}$ (Centro Nacional de Información Biotecnológica). En el caso del gen TCTP, se seleccionó de una biblioteca de tabaco de ADNc (Hai-Tao et al. 2005) porque su papel comienza a ser informado como relevante en el metabolismo de metales (Tabla 1). La RTPCR se realizó utilizando Platinum ${ }^{\circledR}$ SYBR ${ }^{\circledR}$ Green qPCR Super MIX-UDG (Invitrogen), con placas de 48 pocillos. El programa de PCR incluyó un ciclo a $95^{\circ} \mathrm{C}$ durante $15 \mathrm{~min}, 40$ ciclos a $95{ }^{\circ} \mathrm{C}$ durante $15 \mathrm{~min}, 60$ ${ }^{\circ} \mathrm{C}$ durante $60 \mathrm{~min}$, un ciclo a $95{ }^{\circ} \mathrm{C}$ durante $15 \mathrm{~min}$ y $60{ }^{\circ} \mathrm{C}$ durante $60 \mathrm{~min}$. Para el análisis de los datos generados se utilizó una cuantificación comparativa CT $(\Delta \Delta C T)$ utilizando el software StepOne.

\section{RESULTADOS Y DISCUSIÓN}

El análisis de las proteínas solubles totales a diferentes concentraciones de $\mathrm{PbAc}_{2}$ mostró diferencias significativas en las hojas (Figura 1a) y en los tallos (Figura 1b) de las plantas de tomate cv. Mariela. Este parámetro en las hojas fue menor a 50 $\mathrm{mg} \mathrm{kg}^{-1}$ donde hubo $68 \%$ menos proteína que en el 
Tabla 1. Diseño de los cebadores para el PCR en Tiempo Real.

\begin{tabular}{|c|c|c|c|}
\hline & Largo $(\mathrm{pb})$ & $\mathrm{Tm}$ & Secuencia del cebador \\
\hline \multicolumn{4}{|l|}{ SOD } \\
\hline Cebador Izquierdo & 20 & 59.93 & 5' ATGATGGAACTGCCACCTTC 3' \\
\hline $\begin{array}{l}\text { Cebador Derecho } \\
\text { IFR }\end{array}$ & 20 & 59.75 & 5' TAGCAGCTTTAGCCCTGGAG 3' \\
\hline Cebador Izquierdo & 20 & 58.65 & 5' TGCTCAGTACATGTGGGTCA 3' \\
\hline $\begin{array}{l}\text { Cebador Derecho } \\
\text { TCTP }\end{array}$ & 20 & 60.16 & 5' GGATTCTCTTGGCATCCTGA 3' \\
\hline Cebador Izquierdo & 20 & 60 & 5' AGCTGGTCCCTCGTTTTCTT 3' \\
\hline $\begin{array}{l}\text { Cebador Derecho } \\
\text { Ubiquitina }\end{array}$ & 20 & 60 & 5' GCACCTTGAACAACCCACTT 3' \\
\hline Cebador Izquierdo & 21 & 58 & 5' AGAGGAAGGGTTTGAAGCAGG3' \\
\hline Cebador Derecho & 21 & 59 & 5' TTTGCCAGCTTGAACATCCAT3' \\
\hline
\end{tabular}

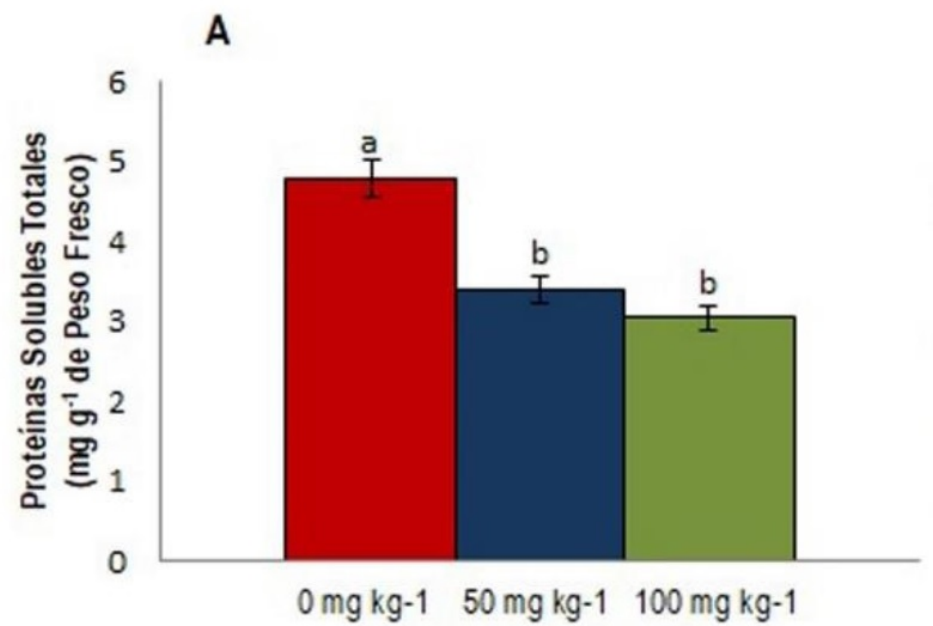

B

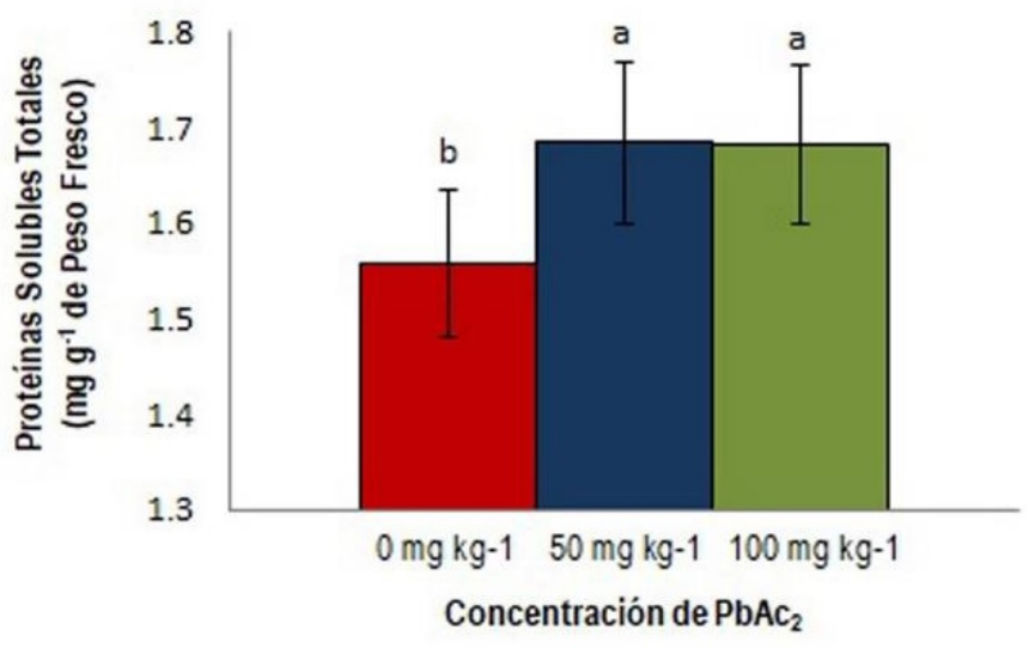

Figura 1. Proteínas Solubles Totales ( $\mathrm{mg} \mathrm{g}^{-1}$ de PF); A) Proteínas en hojas; B) Proteínas en tallos de $S$. lycopersicum cv. Mariela. Letras diferentes indican diferencias significativas $(p \leq 0.05)$. 
control, y a $100 \mathrm{mg} \mathrm{kg}^{-1}$ hubo $58 \%$ menos proteína que en el control bajo la influencia de $\mathrm{PbAc}_{2}$, como se muestra en la Figura 1A. Este resultado podría deberse a que la fotosíntesis se vio afectada, específicamente la máquina fotosintética por su relación con las proteínas. Al respecto Sharma y Dubey (2005) informan que el proceso de fotosíntesis se ve muy afectado por la toxicidad del $\mathrm{Pb}$, además que el contenido de proteínas disminuye cuando se acumula el $\mathrm{Pb}$ (hojas) y a medida que la concentración del metal aumenta (Mohan y Hosetti 1997).

En cuanto al aumento en los tallos de las proteínas solubles totales en las plantas tratadas con $\mathrm{PbAc}_{2}$ esto podría deberse a una saturación en los mecanismos de retención del $\mathrm{Pb}$ en el tallo, teniendo en cuenta que es un órgano de transporte por lo cual su capacidad como reservorio del metal es finita (Abreu et al. 2016).

La actividad de la GR con los tratamientos utilizados fue mayor (133. $3 \%$ ) en hojas a $50 \mathrm{mg} \mathrm{kg}^{-1}$ sin diferencias significativas entre el control y $100 \mathrm{mg}$ $\mathrm{kg}^{-1}$ de $\mathrm{PbAc}_{2}$ (Figura 2a). Los valores encontrados difieren de los informados por Usman et al. (2020) en en Tetraena qatarensis donde la actividad de la GR en toda la planta aumentó con la concentración de $\mathrm{Pb}\left(0,25,50\right.$ y $\left.100 \mathrm{mg} \mathrm{L}^{-1}\right)$. La menor actividad de la GR encontrada en las hojas a $100 \mathrm{mg}$ $\mathrm{kg}^{-1}$ de $\mathrm{PbAc}_{2}$ no difiere significativamente del control pudiendo implicar la inhibición de su actividad a esta concentración. Normalmente en condiciones de estrés la actividad de la enzima debe aumentar como parte de los procesos antioxidantes (Gill et al. 2013). Este resultado coincide con el obtenido por Khan et al. (2020) en plantas de algodón (Gossypium hirsutum L.) tratadas con 0 y $500 \mu \mathrm{M}$ de $\mathrm{Pb}$ donde en las hojas medias no hubo diferencias significativas entre el control y el tratamiento de glutatión (GSH) más $\mathrm{Pb}$.

En los tallos, la actividad de la enzima aumentó un $108.9 \%$ a $50 \mathrm{mg} \mathrm{kg}^{-1}$ y un $105.6 \%$ a $100 \mathrm{mg}$ $\mathrm{kg}^{-1}$ de $\mathrm{PbAc}_{2}$ (Figura 2b). El $\mathrm{Pb}$ en el suelo se mueve desde las raíces hasta las hojas, pasando por el tallo en su camino hacia las hojas; en los tallos la GR comienza su función como enzima antioxidante al eliminar el $\mathrm{H}_{2} \mathrm{O}_{2}$ que se produce principalmente en los cloroplastos para mantener el estado redox de la célula (Asada 1992), lo que pudiera explicar su mayor actividad con las concentraciones de $\mathrm{PbAc}_{2}$ utilizadas.

La actividad de la SOD fue significativamente menor al compararla con el control bajo las dos concentraciones de $\mathrm{PbAc}_{2}$ en ambas partes de la planta estudiadas (Figura 3a, b). La disminución de la actividad de la SOD en presencia de las dos concentraciones de $\mathrm{PbAc}_{2}$ utilizadas y las dos partes de la planta analizadas coincide con los resultados obtenidos en plántulas de manglar (Bruguiera gymnorrhiza) (Tao et al. 2012), esquejes de J. curcas (Shu et al. 2011) y en plantas de cáñamo (Xia et al. 2019). Según Hasanuzzaman et al. (2012), la SOD es la enzima que actúa como la primera línea de defensa porque dismutasa el radical superóxido $\left(\mathrm{O}_{2--}\right)$ convirtiéndolo en $\mathrm{H}_{2} \mathrm{O}_{2}$. La menor actividad de esta enzima en esta investigación podría indicar una disminución de la función de dismutar el radical superóxido. El resultado encontrado en la actividad de la SOD por Machín-Suárez et al. (2017) en el tomate cv. Amalia bajo las mismas concentraciones de $\mathrm{PbAc}_{2}$ fue diferente al resultado de este estudio, lo que indica que en una misma especie de planta las respuestas enzimáticas varían entre cultivares. De manera general la actividad de ambas enzimas en la variedad de tomate Mariela tuvieron un comportamiento diferente pues la GR mostró una mayor actividad en hojas en la concentración de 50 $\mathrm{mg} \mathrm{kg}^{-1}$ de $\mathrm{PbAc}_{2}$ y en tallos aumentó en ambas concentraciones mientras que la SOD disminuyó en ambos órganos y en las dos concentraciones estudiadas. Aunque las dos son enzimas antioxidantes están asociadas con la regulación de las EROs en plantas, cada una tiene una función muy diferente lo que explica este comportamiento. El estrés abiótico provocado por la aplicación de diferentes concentraciones de Plomo, inhibió la actividad enzimática de la SOD y estimuló la actividad enzimática de la GR en hojas y tallos, lo que mejoró el mecanismo de protección basado en la regulación de las actividades enzimáticas y compartimentación de ambas enzimas.

Se analizaron los genes IFR, TCTP y SOD para comprender mejor la respuesta de defensa de la planta contra el estrés oxidativo (Figura 4). La 


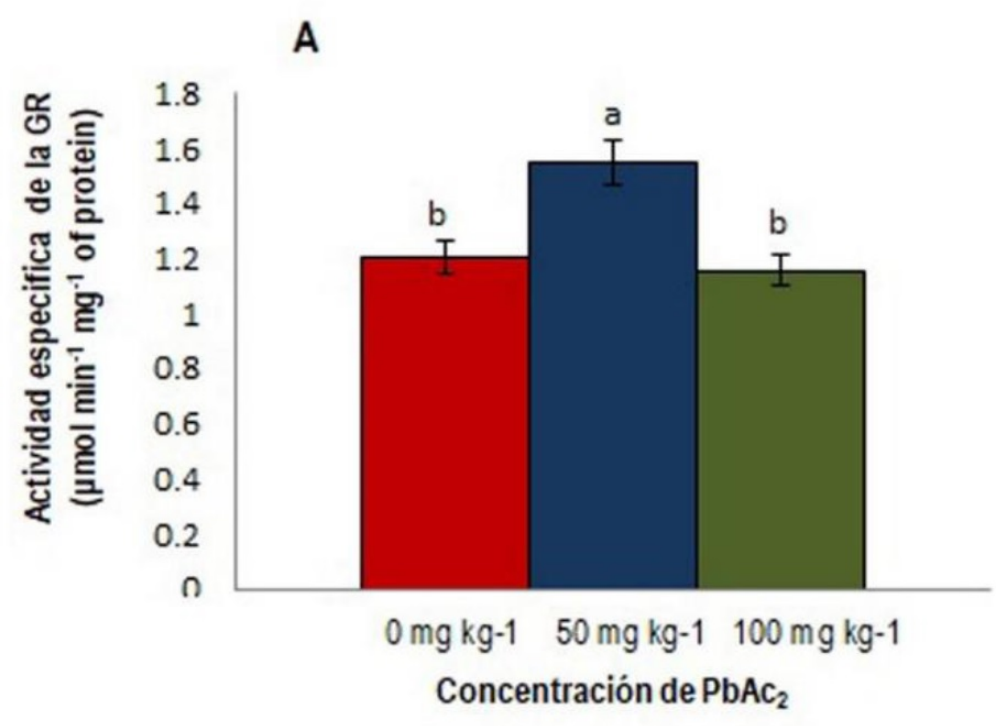

B

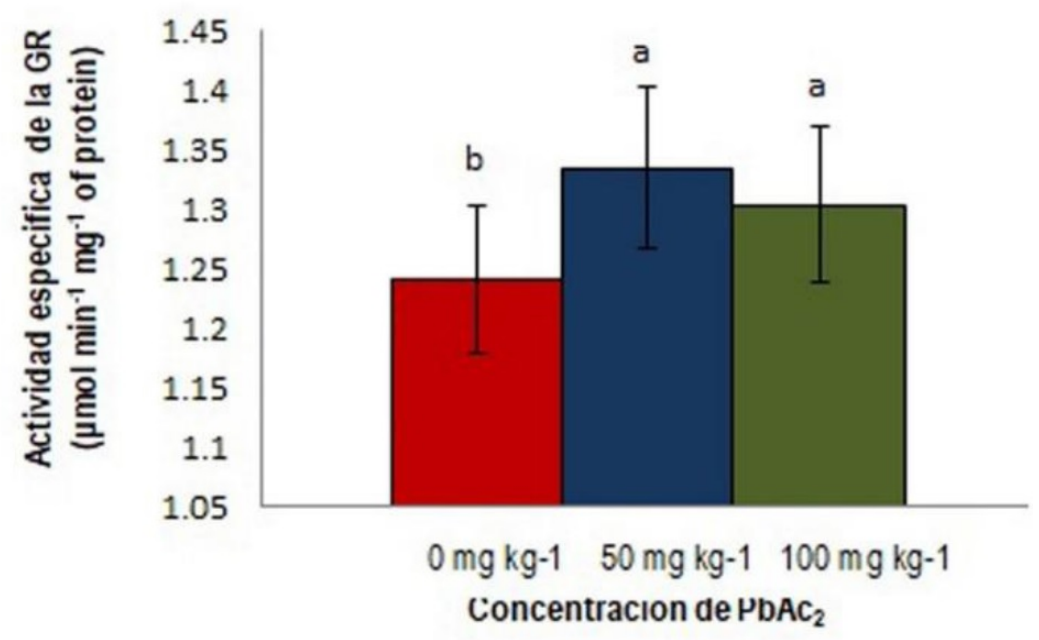

Figura 2. Actividad específica de la enzima GR ( $\mu \mathrm{mol} \mathrm{min}^{-1} \mathrm{mg}^{-1}$ de proteína); A) GR en hojas, B) GR en tallos, de $S$. Iycopersicum cv. Mariela. Letras diferentes indican diferencias significativas $(p \leq 0.05)$.

ubiquitina se utilizó como gen de control debido a su expresión basal estable (Jain et al. 2006). Para todos los genes analizados, la expresión fue mayor bajo estrés por $\mathrm{Pb}$ con diferencias significativas al compararlas con el control. La expresión de la TCTP en hojas en comparación con el control fue $600 \%$ mayor a $50 \mathrm{mg} \mathrm{kg}^{-1}$ y $675 \%$ mayor a $100 \mathrm{mg} \mathrm{kg}^{-1}$ sin diferencias significativas entre los dos niveles de
$\mathrm{PbAc}_{2}$; en los tallos la expresión fue $300 \%$ mayor a $50 \mathrm{mg} \mathrm{kg}^{-1}$ y aumentó $800 \%$ a $100 \mathrm{mg} \mathrm{kg}^{-1}$, en este caso con diferencias significativas entre todos los tratamientos. En plantas, según Bommer (2017), la TCTP parece tener una función protectora frente a estreses fitotóxicos, como la presencia de metales pesados, lo que puede explicar la alta expresión de este gen bajo estrés por $\mathrm{Pb}$ en ambas partes de la planta 


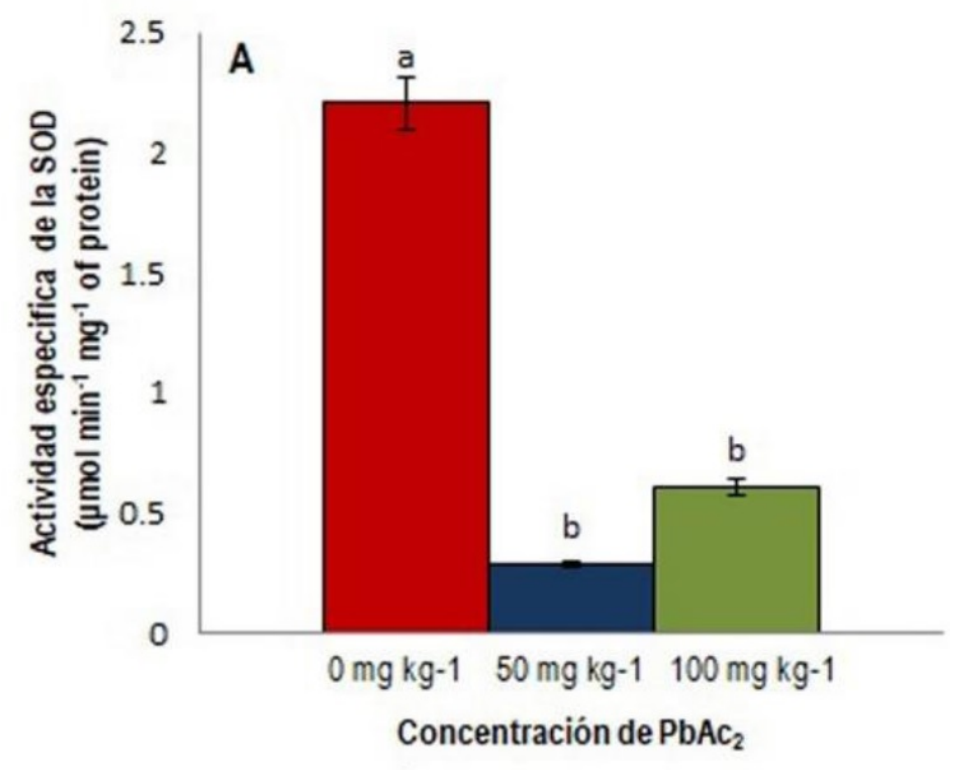

B

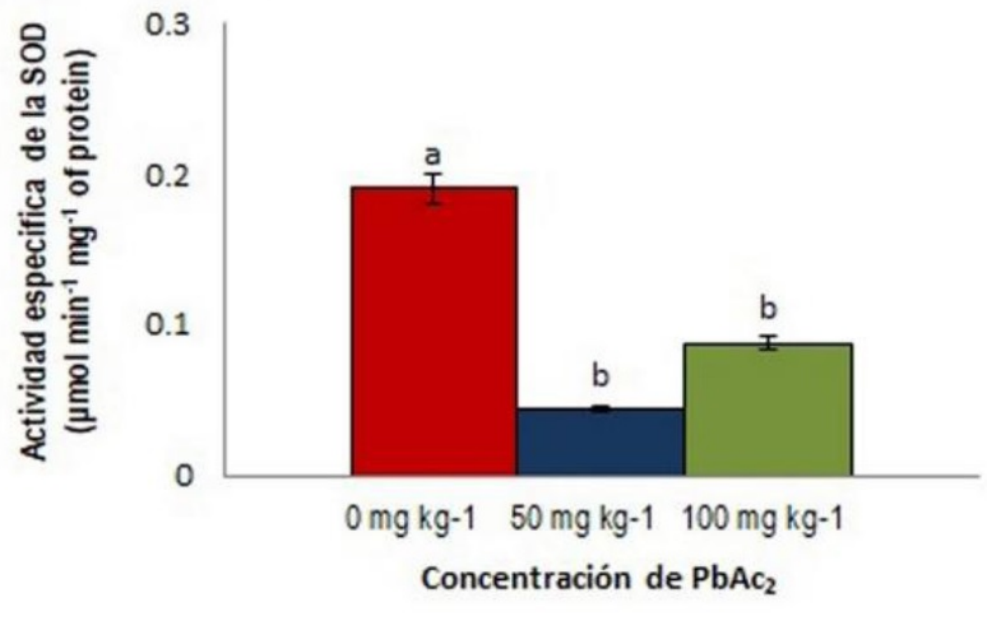

Figura 3. Actividad específica de la enzima SOD ( $\mu \mathrm{mol} \mathrm{min}^{-1} \mathrm{mg}^{-1}$ de proteína); A) SOD en hojas, B) SOD en tallos, de $S$. lycopersicum cv. Mariela. Letras diferentes indican diferencias significativas $(p \leq 0.05)$.

de tomate cv. Mariela. También se ha informado que algunos metales pesados en plantas regulan de forma positiva la TCTP bajo diversas condiciones de estrés celular (Schmidt et al. 2007). En los tallos esta expresión génica no fue diferente entre los tratamientos.

La expresión del gen de la SOD en hojas y tallos fue superior a $50 \mathrm{mg} \mathrm{kg}^{-1}$ de $\mathrm{PbAc}_{2}$ lo cual coincide con lo obtenido en plantas de tomate $\mathrm{cv}$ Micro-Tom donde la expresión de la SOD fue mayor a la menor concentración de $\mathrm{PbAc}_{2}$ (Pérez et al. 2013), y difiere del estudio realizado en plantas de tomate $\mathrm{cv}$. Amalia donde la expresión de la SOD aumentó con las dos concentraciones de $\mathrm{PbAc}_{2}$ utilizadas (MachínSuárez et al. 2017). Los resultados sugieren que el perfil de la transcripción genética no está relacionado con la actividad enzimática bajo estrés por $\mathrm{Pb}$ y que este incremento en la expresión del gen de la SOD pudiera contrarrestar el efecto a nivel bioquímico en cuanto a la disminución de la actividad de la enzima. 


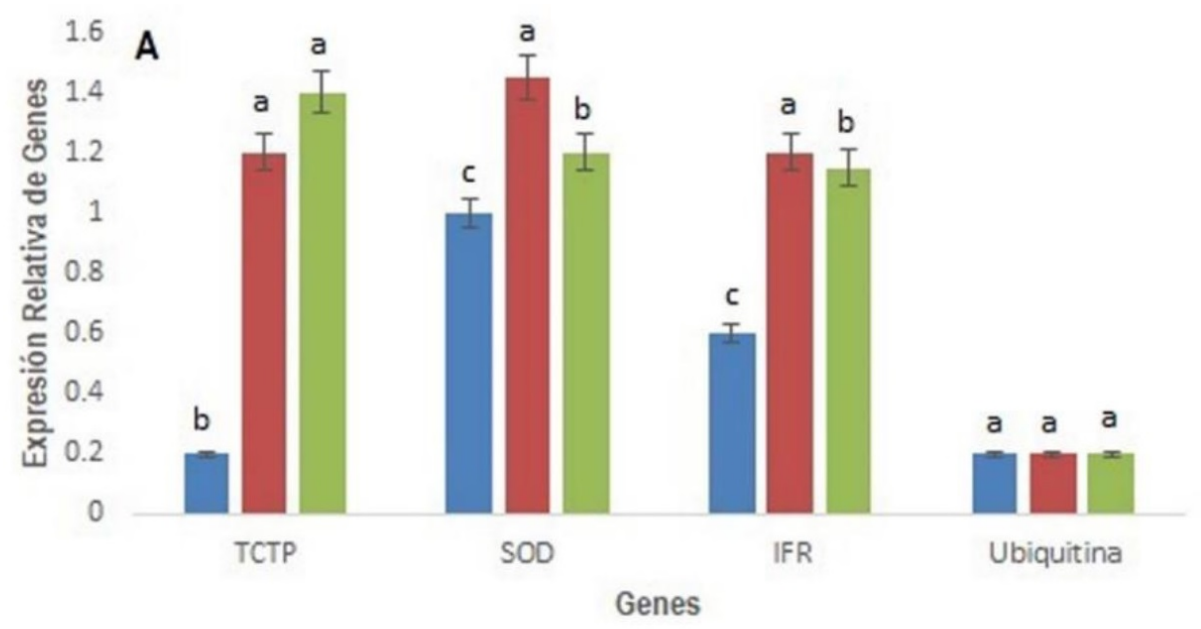

- $0 \mathrm{mg} \mathrm{kg-1}=50 \mathrm{mg} \mathrm{kg-1}=100 \mathrm{mg} \mathrm{kg-1}$

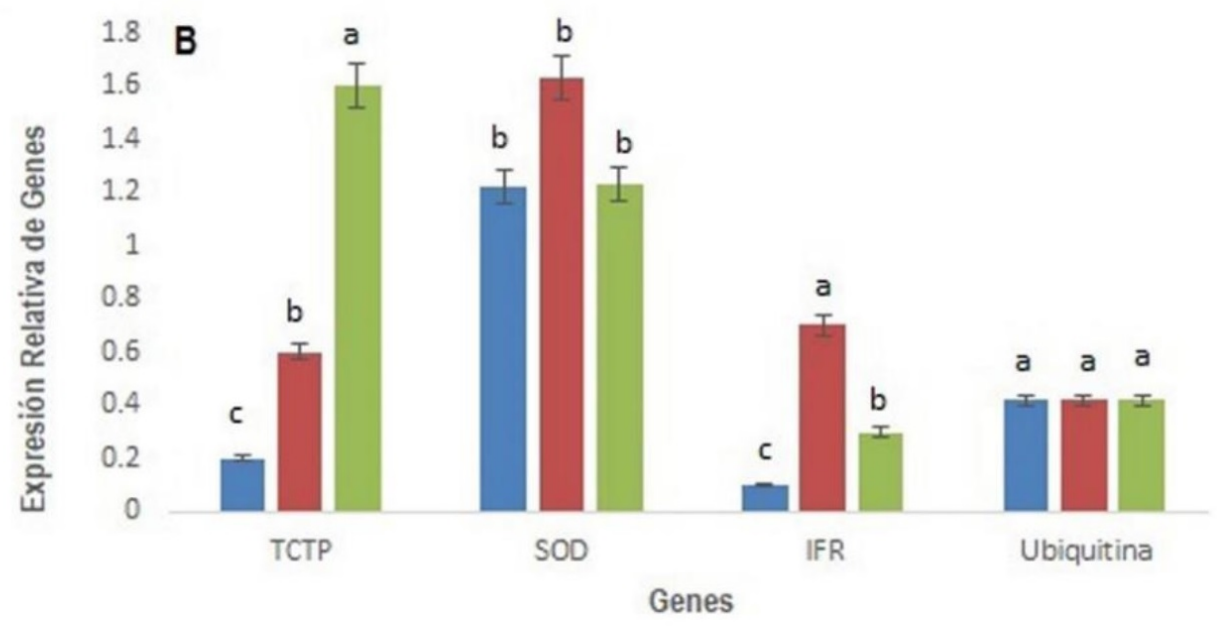

\section{핌 $0 \mathrm{mg} \mathrm{kg-1}=50 \mathrm{mg} \mathrm{kg-1}=100 \mathrm{mg} \mathrm{kg-1}$}

Figura 4. Expresión relativa de la Proteína Tumoral Controlada Transcripcionalmente (TCTP), la Superóxido Dismutasa (SOD) y la Isoflavona Reductasa (IFR) en S. Iycopersicum cv. Mariela, crecida con diferentes concentraciones de $\left.\mathrm{Pb}\left(\mathrm{PbAc}_{2}\right)\left(0,50,10 \mathrm{mg} \mathrm{kg}^{-1}\right), \mathrm{A}\right)$ Hojas, B) Tallos. Letras diferentes indican diferencias significativas $(p \leq 0.05)$.

La expresión del gen IFR fue mayor a $50 \mathrm{mg}$ $\mathrm{kg}^{-1}$ en hojas y tallos en $250 \%$ y $400 \%$ respectivamente. Para el tratamiento de $100 \mathrm{mg} \mathrm{kg}^{-1}$ en hojas aumentó en $240 \%$ y en los tallos $200 \%$ mayor que el testigo con diferencias significativas entre los tratamientos. La expresión del gen IFR fue mayor en hojas y tallos en las dos concentraciones trabajadas de $\mathrm{PbAc}_{2}$, siendo diferente este resultado al obtenido en dos cultivares de arroz (Oryza sativa L.) Hennong 315 y Shendao 47 donde la expresión del gen de la IFR fue baja en ambos cultivares, lo que significa que el estrés por metales pesados disminuyó la expresión del gen (Wang et al. 2020). Resultados similares a los obtenidos en esta investigación fueron informados por Pérez et al. (2013) en plantas de tomate cv-Micro-Tom. La mayor expresión del gen IFR tal vez 
se deba a que los flavonoides pueden funcionar como quelantes de metales (Korkina 2007) y también protegen a las células contra el estrés oxidativo (Rusak et al. 2005). Debido a que hay poca información sobre la expresión del gen IFR en plantas, este trabajo es uno de los pocos que relaciona a este gen con el estrés inducido por $\mathrm{Pb}$.

En plantas de tomate cv. Mariela, la toxicidad del $\mathrm{Pb}$ induce la actividad de la enzima GR, una de las enzimas importantes del sistema de defensa an- tioxidante y reduce la actividad de la SOD en ambas concentraciones de $\mathrm{PbAc}_{2}$ ensayadas. El análisis molecular indica mayor expresión de los tres genes estudiados (SOD, TCTP, IFR) bajo estrés por Pb, lo que sugiere que estos resultados pueden aplicarse en programas de mejoramiento genético de plantas de tomate, para obtener plantas con mayor tolerancia al $\mathrm{Pb}$ y/o con mejores capacidades de acumulación de este metal pesado.

\section{LITERATURA CITADA}

Abreu CB, Sacramento BL, Teixeira AA, Cardim SM, Santos MP, Azevedo AD (2016) Nutritional and biochemical changes induced by lead in sunflower (Helianthus annuus L.). Ciências Agrárias 37: 1229-1242.

Akinci IE, Akinci S, Yilmaz K (2010) Response of tomato (Solanum lycopersicum L.) to lead toxicity: Growth, element uptake, chlorophyll and water content. African Journal of Agricultural Research 5: 416-423.

Al-Qurainy F (2009) Toxicity of heavy metals and their molecular detection on Phaseolus vulgaris (L.). Australian Journal of Basic and Applied Sciences 3: 3025-35.

Asada K (1992) Ascorbate peroxidase-a hydrogen peroxide scavenging enzyme in plants. Physiologia Plantarum 85: 235-241.

Azevedo RA, Alas RM, Smith RI, Lea PI (1998) Response of antioxidant enzymes to transfer from elevated carbon dioxide to air and ozone fumigation, in the leaves and roots of wild type and a catalase deficient mutant of barley. Physiologia Plantarum 104: 280-292.

Beyer W, Fridowich FI (1987) Assaying for superoxide dismutase activity: Some large consequences of minor changes in conditions. Analytical Biochemistry 161: 559-566.

Bizily SP, Rugh CL, Summers AO, Meagher RB (1999) Phytoremediation of methyl mercury pollution: MerB expression in Arabdopsis thaliana confers resistance to organomercurials. Proceedings of the National Academy of Sciences of the United States of America 96: 6808-6813.

BMJV (2017) Federal Administration of Justice and Consumer Protection. Federal Soil Protection and Contaminated Sites Ordinance of July 12, 1999. https://www.gesetze-im-internet.de/bbodschv/BBodSchV.pdf. Fecha de consulta: 9 February 2021.

Bommer UA (2017) The Translational Controlled Tumour Protein TCTP: Biological functions and regulation. In: Telerman A., Amson R. (eds) TCTP/tpt1 - Remodeling signaling from stem cell to disease. Results and problems in cell differentiation, vol 64. Springer International Publishing. Switzerland. pp: 69-126.

Bradford MM (1976) A rapid and sensitive method for the quantitation of microgram quantities of protein utilizing the principle of protein dye binding. Analytical Biochemistry 72: 248-254.

Costa JM, Heuvelink E (2018) The global tomato industry. In: Heuvelink E (ed) Tomatoes. 2nd. CABI. Glasgow, UK. pp: 1-26.

FAOSTAT (2021) Datos sobre alimentación y agricultura. http://www.fao.org/faostat/es/\#data/QC. Fecha de consulta: 9 February 2021. 
Gill SS, Anjum NA, Hasanuzzaman M, Gill R, Trivedi DK, Ahmad I, Pereira E, Tuteja N (2013) Glutathione and glutathione reductase: a boon in disguise for plant abiotic stress defense operations. Plant Physiology and Biochemistry 70: 204-212.

Gomes-Junior RA, Moldes CA, Delite FS, Gratão PL, Mazzafera P, Lea PJ, Azevedo RA (2006) Nickel elicits a fast antioxidant response in Coffea arabica cells. Plant Physiology and Biochemistry 44: 420-429.

Hai-Tao D, Wenzheng L, Pérez SA, Hongmei L, Ye D, Qingchao JCH, Cabezas MD, Yongqi F, Shao J, Lou Y, Shi W, Shao Y, Li Y, Chen J, De-Bao L (2005) Large-scale identification of ESTs from Nicotiana tabacum L. by normalized cDNA library sequencing. Medical Hypotheses and Research 2: 418-496.

Hasanuzzaman M, Hossain MA, da Silva JA, Fujita M (2012) Plant responses and tolerance to abiotic oxidative stress: Antioxidant defense is a key factor. In: Bandi V, Shanker AK, Shanker C, Mandapaka M (eds) Crop stress and its management: Perspectives and strategies. Springer. Berlin, Germany. pp: 261-316.

Jain M, Nijhawan A, Tyagi AK, Khurana JP (2006) Validation of housekeeping genes as internal control for studying gene expression in rice by quantitative real-time PCR. Biochem Biophys. Research Communications 345: 646-51.

Kaur N, Kaur J, Grewal SK, Singh I (2019) Effect of heat stress on antioxidative defense system and its amelioration by heat acclimation and salicylic acid pre-treatments in three pigeon pea genotypes. Indian Journal of Agricultural Biochemistry 32: 106-110.

Khan M, Samrana S, Zhang Y, Malik Z, Daud MK, Zhu Sh (2020) Reduced glutathione protects subcellular compartments from $\mathrm{Pb}$-induced ROS injury in leaves and roots of upland cotton (Gossypium hirsutum L.). Frontier of Plant Science 11: 412. DOI: 1010.3389/fpls.2020.00412.

Korkina LG (2007) Phenylpropanoids as naturally occurring antioxidants: From plant defense to human health. Cellular and Molecular Biology 53: 15-25.

Kumar N, Soni H, Kumar RN, Bhatt I (2009) Hyperaccumulation and mobility of heavy metals in vegetable crops in India. Journal of Agriculture and Environment 10: 29-38.

Machín-Suárez A, Chávez-Sánchez E, Héctor-Ardisana EF, Santos-Cervantes ME, Pérez-Álvarez S (2017) Actividad de enzimas del estrés oxidativo en plantas de tomate cv. Amalia en respuesta al plomo. Horticultura Brasileira 35: 216-223.

Mohan BS, Hosetti BB (1997) Potential phytotoxicity of lead and cadmium to Lemna minor grown in sewage stabilization ponds. Environmental Pollution 98: 233-238.

Moya C, Álvarez M, Caballero A (2000) Evaluación de nuevas líneas de tomate (Lycopersicon esculentum Mill) considerando los criterios de los productores en la metodología utilizada. Cultivos Tropicales 21: 75-79.

Pérez SA, Ahmed IS, Cabezas DM (2013) Molecular and biochemical characterization in tomato (Solanum lycopersicum L.) plants cv. Micro-Tom related to lead (Pb)-induced stress. Revista Biotecnología Aplicada 30: 194-198.

Rusak G, Gutzeit H (2005) Ludwig-Müller J Structurally related flavonoids with antioxidative properties differentially affect cell cycle progression and apoptosis of human acute leukemia cells. Nutrition Research 25: 143-155.

Sachdev S, Ansari SA, Ansari MI, Fujita M, Hasanuzzaman M (2021) Abiotic Stress and Reactive Oxygen Species: Generation, Signaling, and Defense Mechanisms. Antioxidants 10: 1-37. DOI: 10.3390/antiox10020277. 
Schmidt I, Fahling M, Nafz B, Skalweit A (2007) Thiele BJ. Induction of translationally controlled tumor protein (TCTP) by transcriptional and post-transcriptional mechanisms. FEBS Journal 274: 5416-24.

Sharma P, Dubey RS (2005) Lead toxicity in plants. Brazilian Journal of Plant Physiology 17: 3552.

Shu X, Yin LY, Zhang QF, Wang WB (2011) Effect of Pb toxicity on leaf growth, antioxidant enzyme activities, and photosynthesis in cuttings and seedlings of Jatropha curcas L. Environmental Science and Pollution Research 19: 893-902.

Tao YM, Chen YZ, Tan T, Liu XC, Yang DL, Liang SC (2012) Comparison of antioxidant responses to cadmium and lead in Bruguiera gymnorrhiza seedlings. Biologia plantarum 56: 149-152.

Usman K, Abu-Dieyeh MH, Zouari N, Al-Ghouti MA (2020) Lead (Pb) bioaccumulation and antioxidative responses in Tetraena qatarense. Scientific Reports 10: 1-10. DOI: 10.1038/s41598-020-73621-z.

Wang Q, Zeng X, Song Q, Sun Y, Feng Y, Lai Y (2020) Identification of key genes and modules in response to Cadmium stress in different rice varieties and stem nodes by weighted gene co-expression network analysis. Scientific Reports 10: 9525. DOI: 10.1038/s41598-020-66132-4.

Xia Ch, Hong L, Yang Y, Yanping X, Xing H, Gang D (2019) Protein changes in response to lead stress of leadtolerant and lead-sensitive industrial hemp using SWATH technology. Gene 10: 396-412. 